\section{Fungal infections of the cornea}

Department of Microbiology Institute of Ophthalmology Joseph Eye Hospital Tiruchirapalli, India

Correspondence:

PA Thomas

Institute of Ophthalmology

Joseph Eye Hospital

PB 138 Tiruchirapalli

620001, India

Tel: + 914312460622

Fax: + 914312414969

E-mail: philipthomas@

satyam.net.in

Received: 28 February 2003 Accepted in revised form: 28 February 2003

Proprietary interests: Nil

Presented at the Cambridge Ophthalmological

Symposium, 4-6 September 2002

\begin{abstract}
Purpose To describe key aspects of fungal infections of the cornea, which constitute an important eye problem in outdoor workers in tropical and subtropical regions.

Methods Review of published studies and personal observations.

Observations Fungal infections of the cornea are frequently caused by species of Fusarium, Aspergillus, Curvularia, and Candida. Trauma is the most important predisposing cause; ocular and systemic defects and prior application of corticosteroids are also important risk factors. Culture remains the cornerstone of diagnosis; direct microscopic detection of fungal structures in corneal scrapes or biopsies permits a rapid presumptive diagnosis. A variety of antifungals have been evaluated in therapy of this condition. Natamycin can only be given topically, while amphotericin B, miconazole, ketoconazole, itraconazole, and fluconazole can be administered by various routes. Topical amphotericin B $(0.1-0.3 \%)$ is frequently the treatment of choice for infections due to Candida and related fungi, while topical natamycin $(5 \%)$ is the choice for keratitis due to filamentous fungi. Medical therapy may fail, necessitating surgical intervention. Conclusions Fungal infections of the cornea continue to be an important cause of ocular morbidity, particularly in the agricultural communities of the developing world. A proper understanding of agent and host factors involved in these infections will improve the outcome of this condition. Eye (2003) 17, 852-862. doi:10.1038/ sj.eye. 6700557
\end{abstract}

Keywords: corneal infections; keratitis; fungal; mycotic; Fusarium; Aspergillus

Fungal infections of the cornea (mycotic or fungal keratitis, keratomycosis) present as suppurative, usually ulcerative, lesions. Such a corneal infection poses a challenge to the ophthalmologist because of its tendency to mimic other types of stromal inflammation, and because its management is restricted by the availability of effective antifungal agents and the extent to which they can penetrate into corneal tissue.

Mycotic keratitis has been reported from many different parts of the world, ${ }^{1,2}$ particularly tropical areas, where it may account for more than $50 \%$ of all ocular mycoses, ${ }^{3}$ or of all cases of microbial keratitis. ${ }^{4}$ Two basic forms have been recognised: that due to filamentous fungi (especially Fusarium and Aspergillus), which commonly occurs in tropical and subtropical zones, and keratitis due to yeast-like and related fungi (particularly Candida). ${ }^{5}$

Keratitis due to filamentous fungi is believed to usually occur following trauma, the key predisposing factor, in healthy young males engaged in agricultural or other outdoor work; traumatising agents of plant or animal origin (even dust particles) either directly implant fungal conidia in the corneal stroma or abrade the epithelium, permitting invasion by exogenous fungi. Environmental factors (humidity, rainfall, wind) greatly influence the occurrence of filamentous fungal keratitis and may also determine seasonal variations in frequency of isolation and types of fungi isolated. ${ }^{6-9}$ Less frequent predisposing factors include immunological incompetence, prior administration of corticosteroids or antibacterial agents, 'allergic conjunctivitis', and the use of hydrophilic contact lenses.,10-12 It is not known whether the use of traditional eye medicines specifically predisposes to filamentous fungal keratitis. ${ }^{1}$ Conversely, in keratitis due to Candida albicans and related fungi, there is usually some pre-existing ocular (eg insufficient tear secretion, defective eyelid closure) or systemic (eg diabetes mellitus, immunosuppression) condition; this type of keratitis may also supervene on a pre-existing epithelial defect because of herpes keratitis or abrasions caused by contaminated contact lenses. ${ }^{5,13}$

Gopinathan et $a l^{14}$ recently reviewed the epidemiological features of 1352 patients with culture-proven mycotic keratitis seen over a period of 10 years at a tertiary care eye hospital 
in southern India; keratitis due to yeast-like fungi occurred in only nine $(0.7 \%)$ patients, hence the observations made relate overwhelmingly to filamentous fungal keratitis. Males were significantly more frequently affected than females (a ratio of 2.5:1), and $64 \%$ of patients were in the age group 16-49 years. Ocular trauma predisposed to infection in $54 \%$ of patients; trauma occurred significantly more frequently in those working outdoors than in those who were indoors. Ocular factors (particularly prior ocular surgery) and systemic factors (particularly diabetes mellitus) were believed to be significant risk factors in 11 and $8 \%$ of patients respectively. ${ }^{14}$

Trauma by vegetable material is believed to be a specific risk factor for a fungal infection of the cornea; ${ }^{5}$ in two recent series of patients, plant material was reported to be the most frequent traumatising agent in $14-17 \%$ of patients. ${ }^{10,14}$ However, in another series, trauma by vegetable material was found to predispose as frequently to bacterial keratitis as to fungal keratitis; instead, trauma by mud or dust particles was reported significantly more frequently by patients with culture-proven mycotic keratitis than by those with culture-proven bacterial keratitis (Thomas et al, unpublished observations).

More than 105 species of fungi, classified in 56 genera, have been reported to cause oculomycosis. ${ }^{15}$ However, species of Fusarium, Aspergillus, and other hyaline hyphomycetes, and species of Curvularia and other dematiaceous hyphomycetes, are the usual isolates from patients with filamentous fungal keratitis, while Candida albicans is the most frequent cause of keratitis due to yeast-like and related fungi (Table 1).

Fungal infections of the cornea need to be promptly recognised to facilitate a complete recovery. Symptoms are usually nonspecific, although possibly more prolonged in duration (5-10 days) than in bacterial corneal ulcers. Pre-existing ocular or systemic disease needs to be identified for correction.

Table 1 Frequent isolates in fungal infections of the cornea

\section{A. Hyaline filamentous fungi \\ Fusarium (F. solani, F. oxysporum) \\ Aspergillus (A. fumigatus, A. flavus) \\ Scedosporium (S. apiospermum) \\ Penicillium (P. spinulosum, P. citrinum) \\ Acremonium (A. potronii, A. kiliense)}

B. Dematiaceous filamentous fungi

Curoularia (C. lunata, C. geniculata, C. senegalensis)

Bipolaris (B. spicifera, B. hawaiiensis)

Exserohilum (E. rostratum, E. longirostrata)

Coelomycetes (Lasiodiplodia, Colletotrichum)

C. Yeast-like and related fungi

Candida (C. albicans)
In filamentous fungal keratitis, which may involve any part of the cornea, firm (sometimes dry) elevated slough, 'hyphate' lines extending beyond the ulcer edge into the normal cornea, multifocal granular (or feathery) greywhite 'satellite' stromal infiltrates, 'immune ring', Descemet's folds, and mild iritis may be seen ${ }^{1,10}$ (Figures 1 and 2). An endothelial plaque and hypopyon may occur after the first week. ${ }^{9}$ Each case of fungal keratitis, while exhibiting these basic features, may differ from others depending on the aetiological agent. ${ }^{16}$ Fusarium solani keratitis may completely destroy an eye in a few weeks, since the infection is usually severe, and perforation, deep extension and malignant glaucoma may supervene. ${ }^{4,10,16,17}$ Aspergillus species are believed to cause a less severe and not so rapidly progressive form of keratitis, which is more amenable to therapy than Fusarium keratitis; ${ }^{16}$ however, some studies ${ }^{10,17}$ have revealed that $42-60 \%$ of corneal infections due to Aspergillus species lead to keratoplasty, in comparison to 23-32\% of corneal ulcers due to Fusarium species.

Keratitis due to Curvularia species usually presents as superficial feathery infiltration, with only slow

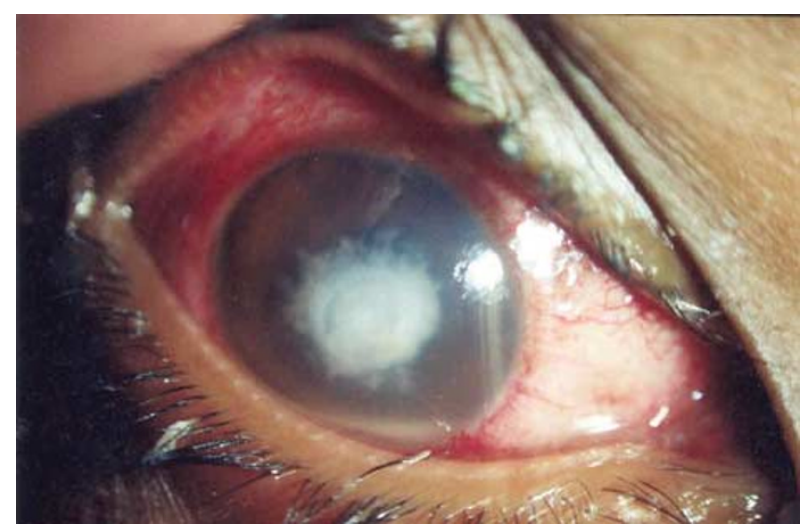

Figure 1 Fungal corneal ulcer of the right eye, exhibiting serrated ('hyphate') margins.

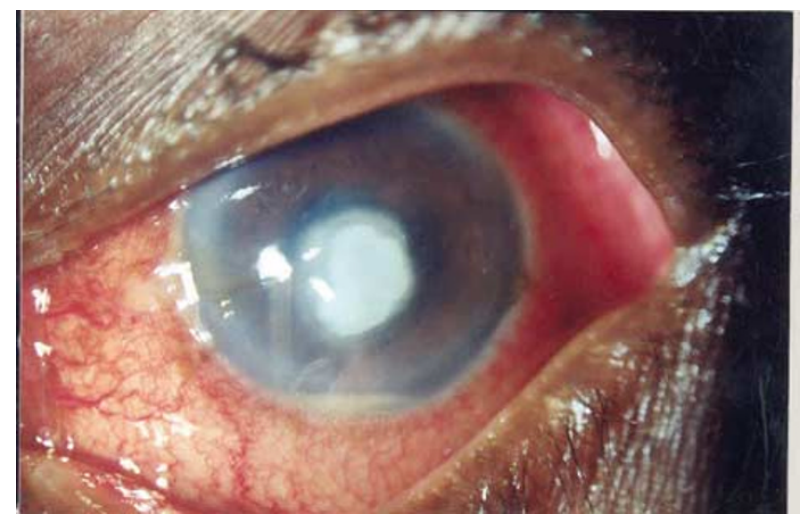

Figure 2 Fungal corneal ulcer of the left eye, exhibiting raised necrotic slough. 
progression to focal suppuration, and topical application of natamycin leads to clinical resolution in most patients; complications requiring surgery, such as exophytic inflammatory fungal sequestration or perforation, are not common, ${ }^{9}$ unless such fungal ulcers are neglected or augmented by steroids. Keratitis due to Scedosporium apiospermum (Pseudallescheria boydii) resembles other types of fungal keratitis in predisposing factors and clinical features but may not be amenable to amphotericin B. Chronic, severe filamentous fungal keratitis may resemble bacterial suppuration and may involve the entire cornea. The stromal keratitis caused by C. albicans and related fungi resembles bacterial keratitis. $^{18}$

Microbiological investigations should always be performed when fungal infections of the cornea are suspected. ${ }^{19}$ Material for microscopy and culture is obtained by scraping the base and edges of the ulcer with a sterile blade or spatula several times. Sometimes, it may not be possible to obtain corneal scrapes because of the occurrence of a very small or nonexistent epithelial defect. In such situations, corneal material may be obtained by performing a corneal biopsy; ${ }^{20,21}$ corneal material may also be obtained at the time of performing a penetrating keratoplasty. ${ }^{10,17}$

Corneal material is inoculated onto several agar (solid) plates (blood agar, brain-heart infusion agar, cystine tryptone agar, Sabouraud glucose-neopeptone agar) in the form of ' $\mathrm{C}$ ' streaks; only growth occurring on the ' $\mathrm{C}$ ' streaks is considered to be significant. Corneal material is also inoculated into broth (liquid) media (Sabouraud broth, brain-heart infusion broth, thioglycollate broth). These culture media can be made more selective for isolation of fungi by addition of antibacterials (gentamicin, chloramphenicol). The media need to be incubated at 30 and $37^{\circ} \mathrm{C}$; the use of liquid-shake cultures is also advisable. ${ }^{15}$ Although fungal growth usually

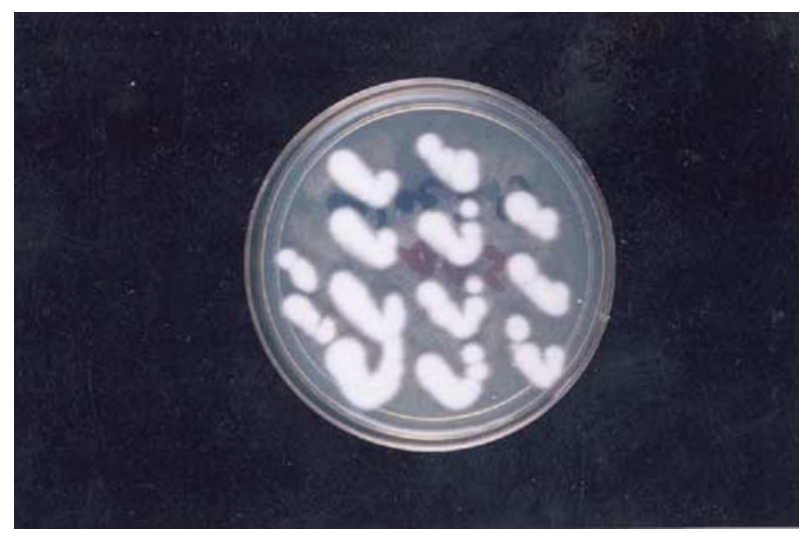

Figure 3 Growth of Fusarium solani on Sabouraud glucoseneopeptone agar plate after $72 \mathrm{~h}$ incubation; the corneal scrapes had been inoculated as ' $\mathrm{C}$ '-streaks.

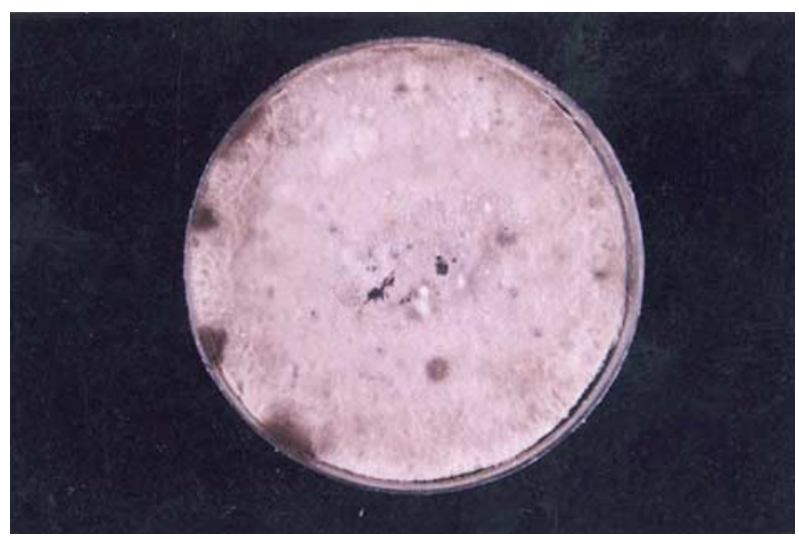

Figure 4 Abundant and rapid growth of Lasiodiplodia theobromae on Sabouraud glucose-neopeptone agar plate after $96 \mathrm{~h}$ incubation.

occurs within 3-4 days (Figures 3,4), culture media may require incubation for upto 4-6 weeks. Growth of fungi in culture is deemed significant if this correlates with the clinical presentation, if growth of the same fungus is demonstrated on two or more solid culture media, or if there is semiconfluent growth at the site of inoculation on one solid medium, or growth in the liquid media, consistent with microscopy; if possible, repeat scrapings should be performed.

Several techniques for direct microscopic examination of corneal scrapings ${ }^{22}$ permit a rapid presumptive diagnosis of mycotic keratitis (Table 2). A suggested set of smears for direct microscopic detection of fungal structures in corneal material would be: a wet preparation (potassium hydroxide $10 \%(\mathrm{KOH})$, or one of its modifications, or lactophenol cotton blue); a smear stained by the Gram or Giemsa method; a smear stained by a special fungal stain (periodic acid Schiff (PAS); Gomori methenamine silver (GMS), calcofluor white $(\mathrm{CW}))^{13,23,24}$ (Figures 5-8). The corneal material should be spread out as thinly as possible.

Culture of corneal biopsies may sometimes yield better results than those of corneal scrapings, ${ }^{20,21,25,26}$ especially if there is active proliferation of fungi in the depth of the corneal stroma with only a small epithelial defect. Direct examination of corneal biopsies or corneal buttons yields positive results more frequently than do fungal cultures of the same specimens, both in experimental animals ${ }^{27}$ and in patients. ${ }^{10,17}$ Fungal structures in sections of corneal tissue can be stained by PAS or GMS stains, ${ }^{28}$ or by fluorochromes, such as CW and fluoresceinconjugated lectins; ${ }^{13,29}$ a peroxidase-labelled lectin staining technique for diagnosis of fungal keratitis due to C. albicans, A. fumigatus, and F. solani in an experimental rabbit model has also been described. ${ }^{30}$

In patients reluctant to undergo corneal biopsy, an impression debridement procedure can be considered; ${ }^{31}$ 
Table 2 Important direct microscopic techniques in fungal infections of the cornea

Method
A. Potassium hydroxide (KOH)
wet mounts
(i) $\mathrm{KOH}$ only
(ii) Ink-KOH
(iii) $\mathrm{KOH}$-dimethyl sulphoxide digestion
with counterstaining (periodic acid-Schiff (PAS),
acridine orange (AO))
Features

A. 1. These mounts require only 1-2 steps to prepare and are inexpensive

2. Use of $\mathrm{KOH}$ ensures good digestion of even thick corneal samples

3. Use of ink, PAS, or AO improves contrast of fungal structures against background

4. Sensitivities of 75-90\% reported for $\mathrm{KOH}$ mounts in culture-proven mycotic keratitis in several Indian studies

5. Reported drawbacks: artefacts common; corneal cells may not swell sufficiently to produce transparent preparations; optimal viewing time for ink-KOH mounts is $12-18 \mathrm{~h}$; ink-KOH has a short shelf-life (ink precipitates out); UV microscope needed if AO counterstain is used

B. Gram staining. Sensitivity of $45-73 \%$

C. Lactophenol cotton blue (LPCB) staining. Sensitivity of $70-80 \%$

D. Methenamine silver staining (modified). Sensitivity of $89 \%$

\section{E. Calcofluor white. Sensitivity of $80-90 \%$}

B. 1. Stains yeast cells and fungal hyphae equally well, and bacteria in the preparation can be differentiated

2. Takes only 5 min to perform

3. Reported drawbacks: may sometimes stain fungal hyphae irregularly or not at all; less useful in thick preparations; false-positive artefacts common; crystal violet precipitates may cause confusion

C. 1. Rapid, simple, inexpensive one-step method which detects all common ocular fungi and Acanthamoeba cysts

2. Stain commercially available and has long shelf-life; LPCB can be kept for years.

3. Reported drawbacks: no tissue digestion, hence thick preparations may pose problems; contrast between fungi and background may be insufficient; unusual fungi may escape detection

D. 1. Fungal cell walls and septa clearly delineated against pale green background

2. Acanthamoeba cysts and Pneumocystis carinii can also be detected

3. Reported drawbacks: excessive deposition of silver may obscure details; staining of cellular debris and melanin may give false-positive results; procedure has multiple steps and takes $60 \mathrm{~min}$; reagents and procedures need standardisation

E. 1. Fungal hyphae and yeast cells clearly delineated against dark background; clearly seen even in thick preparations

2. Acanthamoeba cysts and Pneumocystis carinii can also be detected

3. Two-step method

4. Reported drawbacks: all reagents need to be fresh, otherwise false-positive artefacts occur; ultraviolet microscope needed; reagents and procedures need standardisation; viewer should be protected against hazards of ultraviolet light

Modified from Thomas et al. ${ }^{2}$ Also incorporates observations from references 7,10,14,15,22-25,27

here, a cellulose acetate filter paper (of the type used for conjunctival impression cytology) is gently applied to the ulcerated part of the cornea to obtain a 'replica', and this is subsequently stained and examined.

The polymerase chain reaction (PCR) is a novel technique for amplification of DNA in tissue samples. This technique was applied to the diagnosis of experimental keratitis due to $F$. solani in rabbits. ${ }^{32}$ Amplified target DNA sequences were noted in $89 \%$ of 28 samples from Fusarium-infected corneas, whereas only $21 \%$ of 14 samples from infected corneas were positive by culture; however, culture was $100 \%$ specific whereas the
PCR technique was $88 \%$ specific. In a clinical setting, the PCR method was used to establish a diagnosis of fungal keratitis caused by Alternaria alternata within $24 \mathrm{~h}$, whereas growth of filamentous fungi in culture was obtained after 6 days and the definitive identification made after 10 days; ${ }^{33}$ since it was too late for medical therapy to succeed in this patient, and penetrating keratoplasty had to be performed, the authors emphasised the advantage provided by PCR compared to conventional culture techniques, in terms of time. In another study, ${ }^{34}$ a PCR-based assay, developed to amplify a part of the fungal $18 \mathrm{~S}$ ribosome gene, was used 


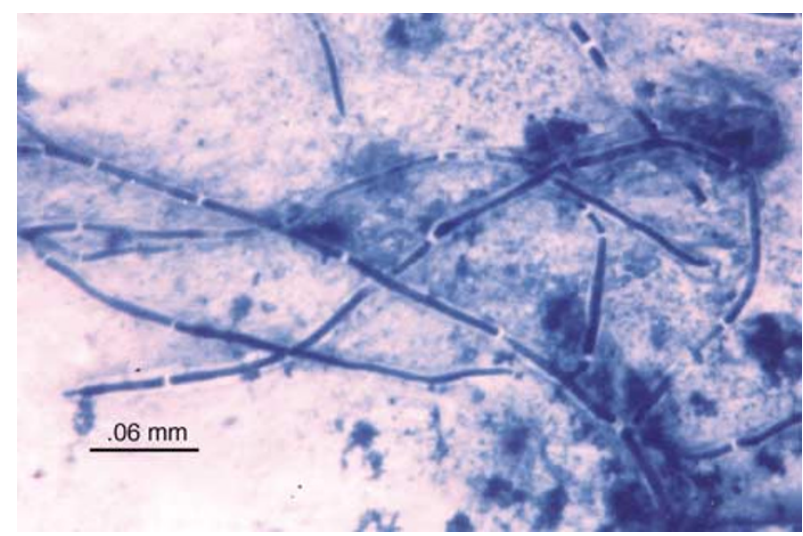

Figure 5 Septate, branching fungal hyphae (cytoplasm stained cell walls and septae unstained) in corneal scrapes; lactophenol cotton blue stain (magnification $\times 400$ ). Bar $=0.06 \mathrm{~mm}$.

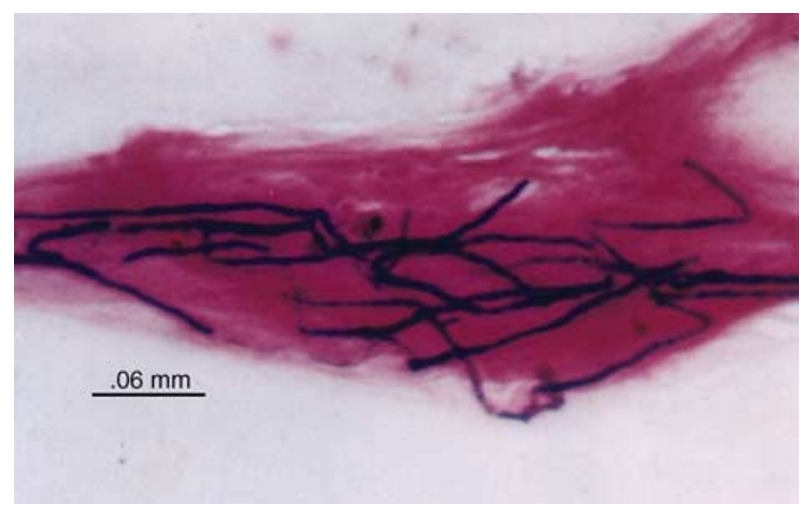

Figure 6 Branching fungal hyphae (cytoplasm stained by crystal violet) in corneal scrapes; Gram stain (magnification $\times 400)$. Bar $=0.06 \mathrm{~mm}$.

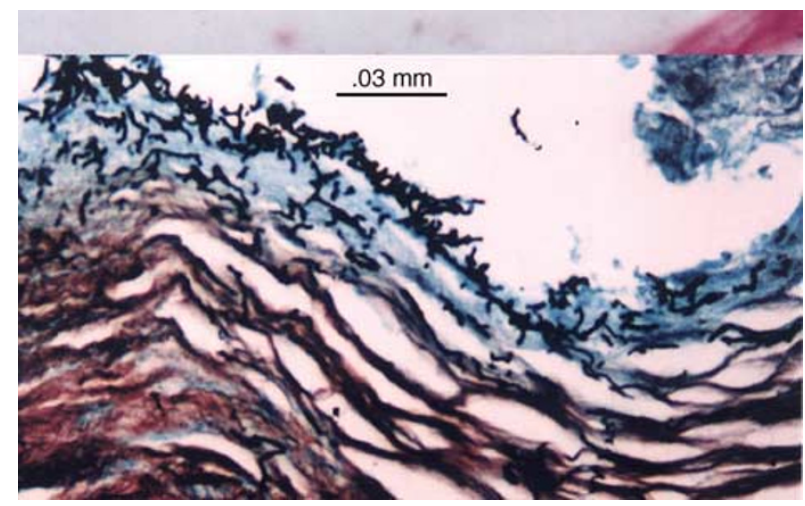

Figure 7 Numerous branching fungal hyphae in superficial stroma of corneal button; tissue section ( $8 \mu \mathrm{m}$ thickness) stained by Gomori methenamine silver (magnification $\times 100$ ). Bar $=0.03 \mathrm{~mm}$.

for detection of fungal DNA in corneal scrapes from 30 patients with presumed infectious keratitis, and in the conjunctival swabs of each patient's healthy fellow eye.

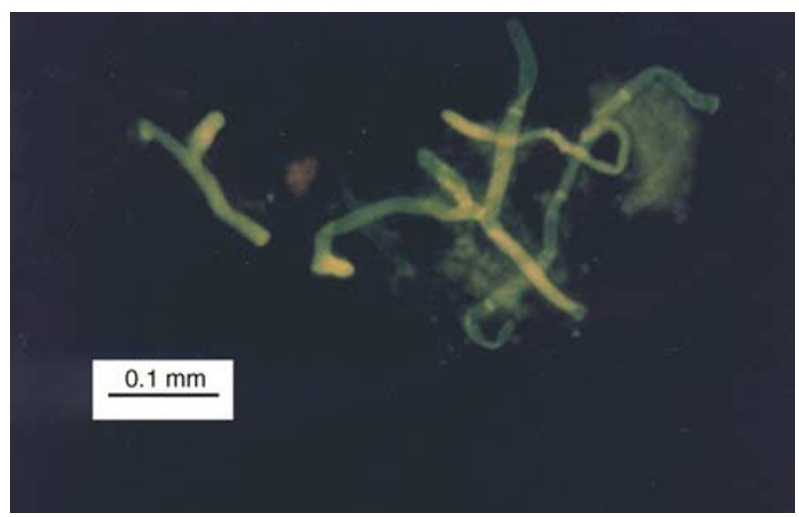

Figure 8 Septate, branching fungal hyphae in corneal scrapes stained by calcofluor white (magnification $\times 1000$ ); hyphae fluoresce bright greenish-yellow. Bar $=0.1 \mathrm{~mm}$.

PCR and fungal culture results matched in $74 \%$ of the corneal scrapes. In relation to culture, the sensitivity of PCR was $94 \%$ but specificity was only $50 \%$; a falsenegative PCR result was obtained in $3 \%$. At present, PCR appears to be promising as a means to diagnose fungal keratitis, offering some advantages over culture methods, including rapid analysis and the ability to analyse specimens far from where collected; however, the possibility of false-positive results needs to be always considered.

Although some workers ${ }^{16,17}$ opine that antifungal susceptibility testing of the fungus isolated from a patient with mycotic keratitis aids the selection of specific antifungal therapy for mycotic keratitis, this is a topic fraught with controversy. This is because many different groups of workers have used varying techniques with few details of standardisation or reproducibility. ${ }^{23}$ The advent of the standardised NCCLS technique ${ }^{35}$ may help to resolve this debate.

Mycotic keratitis is managed by medical or surgical means. Medical therapy consists of nonspecific measures and the use of specific antifungal agents. Cycloplegics are used to relieve the iridocyclitis (anterior uveitis) that usually accompanies mycotic keratitis; broad-spectrum antibacterials may be needed to combat secondary bacterial infection. ${ }^{10,36}$

Efforts have been made to evaluate the efficacy of various antiseptics in therapy of mycotic keratitis, particularly in developing countries where there may be limited access to specific antifungal therapy. The application of swabs impregnated with $5 \%$ povidoneiodine once a minute for 3-5 min to infected corneal ulcers was reported to be totally or partially effective in 34 of 35 infected corneal ulcers; ${ }^{37}$ however, no details were provided about the organisms causing the infection. Polyhexamethylene biguanide, a general environmental biocide that exhibits good in vitro activity against various 
microorganisms, including fungi, in a concentration of $0.02 \%$, was found to significantly reduce fungal growth in a rabbit model of $F$. solani keratitis. ${ }^{38}$ A related compound, chlorhexidine (a bis-biguanide cationic antiseptic) was initially reported to show promising activity in mycotic keratitis in two clinical trials, ${ }^{39,40}$ but not in subsequent studies. In a prospective, randomised, controlled, double-masked clinical study on patients with mycotic keratitis, a higher success rate was reportedly achieved with the use of $1 \%$ silver sulphadiazine ointment than with $1 \%$ miconazole ointment; although the response of keratitis due to Aspergillus spp was comparable in both groups, keratitis due to Fusarium spp responded better to silver sulphadiazine than to miconazole. ${ }^{41}$ These excellent results have not been achieved by other workers.

Before specific antifungal therapy is instituted, it may be useful to grade the severity of the keratitis. ${ }^{5}$ If an ulcer is graded to be nonsevere, investigation and treatment may be deferred for $24 \mathrm{~h}$ to allow a period of 'drug washout', whereas in severe keratitis, investigation and treatment need to be instituted at once. ${ }^{5}$

If direct microscopic examination of corneal scrapes or corneal biopsies yields definite results that are consistent with the clinical picture, treatment may be initiated; otherwise, therapy may need to be withheld until culture reports become available. ${ }^{13}$ The antifungal agent finally selected (Table 3) may depend on availability and other criteria. Treatment may be prolonged since the effective concentrations achieved by most antifungal agents in the cornea, with the possible exception of amphotericin B, only inhibit growth of the fungus, necessitating the eradication of fungi by the host defence mechanisms. ${ }^{42,43}$ Topical natamycin $(5 \%)$ or amphotericin B $0.15 \%$ is usually selected as first-line therapy for superficial keratitis, whether or not septate hyphae or yeast cells have been seen by direct microscopy; the presence of deep lesions necessitates the addition of some form of systemic therapy, such as subconjunctival or intravenous miconazole, oral ketoconazole, oral itraconazole, or oral fluconazole. ${ }^{10,17,36,44}$ If hyphae are definitely seen by microscopy, topical natamycin $(5 \%)$ is the drug of choice $\left(0.15 \%\right.$ amphotericin B is another option). ${ }^{42,45}$ Econazole (1\%) was recommended by some workers in the $1970 s^{16,46}$ but there are no recent reports about the use of this compound as first-line therapy for mycotic keratitis. If yeasts or pseudohyphae are seen on microscopy, topical $0.15 \%$ amphotericin $\mathrm{B},{ }^{10,44}$ fluconazole $2 \%,{ }^{47}$ natamycin $5 \%,{ }^{10}$ other azoles at $1 \%$ in arachis oil, or $1 \%$ flucytosine eye drops can be used. ${ }^{13}$ Topical therapy is usually applied hourly around the clock for several days and the frequency of application is then gradually reduced; 42 'topical saturation therapy' has also been advocated. ${ }^{46}$ Some form of systemic therapy (oral azoles, intravenous miconazole) is usually included for severe keratitis. ${ }^{10,36,44,48-50}$

Topical $2 \%$ fluconazole was found efficacious in six Indian patients with microscopy- and culture-proven Candida keratitis with deep lesions, ${ }^{47}$ three of these patients had not responded to topical natamycin, while the other three had not responded to topical miconazole. In another study in India, a prospective evaluation of the comparative safety and efficacy of topical natamycin and $0.2 \%$ fluconazole in eight patients with filamentous fungal keratitis (five due to Acremonium spp, two Curvularia spp, and one unidentified) was attempted. ${ }^{51}$ The ulcers of three of four patients receiving primary natamycin treatment (mean duration 20 days) healed, while one worsened in spite of topical natamycin and oral itraconazole. All four patients who received topical fluconazole as primary treatment failed to respond; two of these were subsequently cured when treated with natamycin, while the other two did not respond to natamycin. These authors concluded that fluconazole cannot be considered as the agent of choice in therapy of keratitis due to filamentous fungi.

Over a period of 10 years (1984-1994), 330 patients with culture-proven mycotic keratitis seen at this institute received ketoconazole (orally $600 \mathrm{mg} /$ day and/ or topically as a $1 \%$ suspension), itraconazole (orally $200 \mathrm{mg}$ /day and/or topically as a $1 \%$ suspension), amphotericin B (topically as a $0.15 \%$ solution), or natamycin (topically as a $5 \%$ suspension) as initial

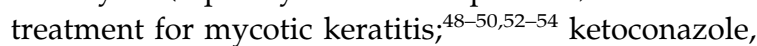
itraconazole, and amphotericin B had to be used for initial treatment because of the nonavailability of natamycin between 1984 and 1990. It was observed that $69 \%$ of patients responded to ketoconazole, $66 \%$ to itraconazole, $53 \%$ to amphotericin B, and $56 \%$ to natamycin; in patients with nonsevere keratitis, $100 \%$ responded to ketoconazole, $86 \%$ to itraconazole, $67 \%$ to amphotericin B, and $84 \%$ to natamycin, while the corresponding figures for severe keratitis were 60, 64, 44, and $28 \%$, respectively. In patients with Fusarium keratitis, $60 \%$ responded to ketoconazole, $54 \%$ to itraconazole, $56 \%$ to amphotericin B, and $44 \%$ to natamycin, while in patients with Aspergillus keratitis, the corresponding figures were $55,73,62$, and 59\% respectively. Curvularia keratitis responded well to all agents. In a more recent evaluation of topical 5\% natamycin for mycotic keratitis, conducted on 246 patients with culture-proven fungal infection over a period of 3 years, $23 \%$ of ulcers were found to have healed, $38 \%$ were healing when last seen, $31 \%$ progressed, while the outcomes in $8 \%$ of patients were not known (Kalavathy and Thomas, unpublished observations). A total of $70 \%$ of nonsevere ulcers and $34 \%$ of severe ulcers had healed or were healing when last seen; this included $62 \%$ of Fusarium ulcers, $56 \%$ of 
Table 3 Principal antifungal drugs used to treat fungal infections of the cornea

Drug, features, and advantages

1. Natamycin (pimaricin)

a. Polyene

b. Commercially available as topical $5 \%$ suspension for ophthalmic use in some countries, where it constitutes first-line therapy for mycotic keratitis

c. Broad spectrum of activity against filamentous fungi and yeast-like fungi causing corneal infections

d. Ophthalmic preparation is well tolerated, stable and can be sterilised by heat

e. Relatively high levels reportedly achieved in cornea after topical application

f. Treatment of keratitis due to species of Fusarium, Aspergillus, Curvularia, and Candida generally yields good results

2. Amphotericin $B$

a. Macrocyclic polyene, which is variably fungistatic or fungicidal

b. Active against Aspergillus spp and Candida spp; emergence of resistant mutants is rare

c. Commonly administered as a topical $0.15-0.30 \%$ solution; intracameral administration $(20-30 \mu \mathrm{g} / \mathrm{ml})$ also reported. Intravenous route may be used in desperate cases

d. Considered as first-line treatment of keratitis due to Candida spp in many countries, and of mycotic keratitis in general in regions where natamycin is not available

e. Penetrates deep corneal stroma after topical application; bioavailability sufficient for susceptible fungi

f. In addition to direct fungicidal effect, also shows immunoadjuvant properties

g. Topical preparation $(0.15 \%)$ is well-tolerated

h. Experimental and clinical data suggest that collagen shields soaked in this drug $(0.5 \%)$ are useful and convenient to treat mycotic keratitis (especially that caused by C. albicans)

3. Flucytosine (5-fluorocytosine)

a. A synthetic fluorinated pyrimidine

b. Topical ( $1 \%$ ) solution well tolerated; can be given orally $(150 \mathrm{mg} / \mathrm{kg} /$ day ) or intravenously

c. Active against Candida, Cryptococcus, and related fungi; effective against some strains of Aspergillus

d. Most useful as adjunctive therapy for yeast keratitis

4. Clotrimazole

a. Imidazole

b. Used topically as $1 \%$ solution or cream; oral route not used now

c. Broad spectrum of activity against Aspergillus in vitro and in vivo; hence was suggested (years ago) as treatment of choice for Aspergillus keratitis

5. Miconazole

a. Synthetic phenylethyl imidazole

b. Reported routes of administration in mycotic keratitis: topical $(1 \%)$, subconjunctival $(10 \mathrm{mg})$, intravenous (600-1200 mg/day); topical and subconjunctival administration generally well tolerated
Drawbacks

a. Not commercially available as an ophthalmic preparation in many regions

b. Effective only when applied topically

c. Natamycin therapy may not be effective when keratitis is associated with deep stromal lesions

d. Only about $2 \%$ of total drug in corneal tissue is bioavailable

a. Fungi such as Scedosporium sp are frequently resistant in vitro; variable activity against Fusarium spp

b. Subconjunctival injection causes marked tissue necrosis at the injection site

c. Very toxic when deoxycholate is used as vehicle

d. Poor intraocular penetration after intravenous administration; this route frequently associated with renal tubular damage

e. Not available as an ophthalmic preparation; for topical use, needs to be reconstituted from the powder or the intravenous preparation

a Cannot be administered alone in treating Candida or Cryptococcus keratitis due to rapid emergence of resistance b. Limited spectrum of activity against filamentous fungi

a. Poorly soluble in water, hence cannot be given parenterally

b. Corneal toxicity associated with long-term use of topical preparation

a. Use of i.v. preparation is occasionally associated with toxicity due to the vehicle used

c. Variable results obtained when treating Fusarium keratitis 
Table 3 (continued)

\begin{tabular}{l} 
Drug, features, and advantages \\
\hline c. Broad spectrum of activity against many ocular pathogenic \\
fungi, including Aspergillus, Candida and Scedosporium \\
d. Once advocated as second-line treatment for \\
keratitis unresponsive to natamycin \\
e. Concomitant administration of oral ketoconazole and \\
topical miconazole found useful in clinical mycotic keratitis
\end{tabular}

6. Econazole
a. Imidazole
b. Topical $(1 \%)$
c. Broad spectrum of activity against filamentous fungi

d. Good results obtained in treating clinical Fusarium keratitis

\section{Ketoconazole}

a. Synthetic dioxolane imidazole

b. Oral (200-600 mg/day) Topical 1-2\%

c. Good in vitro activity against Aspergillus flavus, Candida spp, Curvularia spp, and some other ocular fungal pathogens

d. Reported useful in treatment of nonsevere mycotic keratitis following oral administration

e. Concomitant administration of oral ketoconazole and topical miconazole reported to be useful for clinical mycotic keratitis

8. Itraconazole

a. Synthetic dioxolane triazole

b. Oral capsule (200-400 mg/day); well-absorbed and excellent safety profile after oral administration. A 1-2\% topical suspension in artificial tears. Oral solution and intravenous formulation recently developed, but no reports about use of these for therapy of fungal infections of the cornea

c. Good in vitro activity against all Aspergillus species, Candida, and many dematiaceous fungi

d. No interactions with oral antidiabetic drugs

e. Reported to be very useful for treatment of Aspergillus and Curvularia keratitis, even when severe, and in nonsevere Fusarium keratitis

9. Fluconazole

a. Synthetic bistriazole

b. Oral (200 mg/day) Topical 1-2\% solution Intravenous preparation at $2 \mathrm{mg} / \mathrm{ml}$ (dose is $100 \mathrm{mg} /$ day)

c. Excellent safety profile and good intraocular penetration

d. Increasingly available worldwide; available as topical ophthalmic preparation in some countries

e. Excellent results in natamycin-resistant and miconazole-resistant Candida keratitis with deep lesions

f. Intravenous fluconazole with oral itraconazole and penetrating keratoplasty reported useful for Acremonium keratitis

\section{Drawbacks}


Aspergillus ulcers, and $80 \%$ of Curvularia ulcers. Thus, hourly application of topical $5 \%$ natamycin now constitutes primary therapy for mycotic keratitis at this institute, with addition of oral ketoconazole or itraconazole for severe keratitis.

Since mycotic keratitis usually responds slowly over a period of weeks to antifungal therapy, clinical signs of improvement should be carefully noted: these include diminution of pain, decrease in size of infiltrate, disappearance of satellite lesions, rounding out of the feathery margins of the ulcer and hyperplastic masses or fibrous sheets in the region of healing fungal lesions. ${ }^{42}$ Conjunctival chemosis and injection and punctate epithelial keratopathy may indicate toxicity of the antifungal agent being used. Negative scrapings during treatment do not always indicate that the fungal infection has been eradicated, since there may be active proliferation of the fungi deep in the stroma; hence, therapy should be continued for at least 6 weeks.

Patients with deep corneal infection (perhaps as a result of limited penetration by antifungals) and those who have received corticosteroids before the diagnosis of mycotic keratitis appear to respond less than optimally to antifungal therapy. ${ }^{43}$ Fungal growth in tissue is aided by corticosteroids, which mitigates against their use, alone or in combination with antifungal agents, in mycotic keratitis. ${ }^{43}$ Methods to enhance the efficacy of antifungal agents by administration of several compounds simultaneously also require careful study, since there is the risk of antagonism when combining certain antifungals. $^{42}$

If corneal infection progresses in spite of vigorous antifungal therapy, surgical intervention may be required. Any one or a combination of several methods could be contemplated, depending on the nature, extent, and severity of the corneal infection. However, every effort should be made to prolong medical therapy for the maximum duration possible, to render the infecting fungus nonviable prior to surgery and therein to improve the outcome. Surgery attempts to remove antigenic and/ or infectious elements and also necrotic tissue and other debris, which may hinder complete healing of the lesion. For management of small superficial ulcers, the methods advocated include debridement or pedicle (racquet) conjunctival flaps (for small peripheral ulcers) in association with antifungal therapy; tissue adhesives (n-butyl cyanoacrylate glue) and a bandage contact lens have also been advocated. 5,10,13,55 For more severe ulcers, a Gunderson flap may be tried; however, this procedure is technically difficult to perform since the tissue bleeds profusely, the view of the ulcer is obscured therein precluding monitoring, perforation of the flap and ulcer may occur, the infected material is not removed, and penetration of antifungals may be hindered ${ }^{55}$. Full- thickness corneal grafting (penetrating keratoplasty) is indicated if there is impending perforation, if a perforation exceeding $2 \mathrm{~mm}$ has occurred, or if there is no response to medical therapy. The donor button is usually cut so as to be about $0.5 \mathrm{~mm}$ bigger than the recipient corneal bed. As far as possible, the lens should be left undisturbed to prevent spread of the infection to the posterior segment; however, where the lens is already exposed preoperatively because of a large perforation, lens extraction should be performed through the trephination wound. ${ }^{1,2,13}$

Fungal malignant glaucoma ${ }^{16}$ may occur in a small percentage of patients with mycotic keratitis, especially those with Fusarium infections; this complication is recognised by the occurrence of elevated intraocular tension, uniform shallowing of the anterior chamber, and a fungus-exudate-iris mass covering the pupillary area. Keratoplasty, with a good anterior and posterior chamber wash, an extracapsular lens extraction and postoperative systemic antifungal therapy, is recommended for management, and full dilatation of pupils early in the course of the disease is recommended as prophylaxis; a more invasive procedure, including anterior vitrectomy, may be unnecessary. ${ }^{56}$

Despite advances in diagnosis and medical treatment of fungal infections of the cornea, $15-27 \%$ of patients require surgical intervention owing to failure of medical therapy or advanced disease at presentation. ${ }^{17}$ The outcome of mycotic keratitis ultimately depends on the interplay of agent (invasiveness, toxigenicity, resistance to drugs), host (inflammatory response, hypersensitivity reactions), and predisposing factors. ${ }^{1}$ It has been hypothesised that rapid progression of mycotic keratitis in the early phases is mainly by agent factors, such as a large fungal inoculum and penetration deep into the corneal stroma, while progression in the later phases involves a combination of agent and host factors and resistance to antifungals. ${ }^{17}$ Invasiveness of fungi in corneal tissue may be aided by formation of intrahyphal hyphae, ${ }^{57,58}$ or by liberation of fungal proteinases ${ }^{59}$ or toxins. ${ }^{60}$ Progression of mycotic keratitis may be augmented by the activation of resident corneal cells or inflammatory cells, particularly polymorphonuclear leucocytes, which release proteinases of different molecular weights, resulting in extensive degradation of the corneal tissue matrix. ${ }^{61}$ Current treatment regimens for mycotic keratitis need to be modified to incorporate therapeutic modalities to counter these corneadestroying factors.

\section{Acknowledgements}

I gratefully acknowledge the help rendered by my colleagues Dr CA Nelson Jesudasan, Dr CM Kalavathy, 
Dr J Kaliamurthy, Dr Manuel John, Mr D Arvind Prasanth and Mrs A Geetha in the preparation of this manuscript, and in the studies on fungal infections of the cornea at Joseph Eye Hospital, Tiruchirapalli, India.

\section{References}

1 Thomas PA. Mycotic keratitis: an underestimated mycosis. J Med Vet Mycol 1994; 32: 235-254.

2 Thomas PA, Geraldine P, Kaliamurthy J. Current perspectives in mycotic keratitis: diagnosis, management and pathogenesis. In: Srivastava OP, Srivastava AK, Shukla PK (eds). Advances in Medical Mycology, Vol 2. Evoker Research Perfecting: Lucknow, India, 1997, pp 111-131.

3 Srinivasan R, Kanungo R, Goyal JL. Spectrum of oculomycosis in South India. Acta Ophthalmol 1991; 69: 744-749.

4 Sharma S, Srinivasan M, George C. The current status of Fusarium species in mycotic keratitis in south India. Indian J Med Microbiol 1993; 11: 140-147.

5 Jones DB. Strategy for the initial management of suspected microbial keratitis. In: Barraquer JI, Binder PS, Buxton JN, Fine M, Jones DB, Laibson PR et al (eds). Symposium on Medical and Surgical Diseases of the Cornea. Transactions of the New Orleans Academy of Ophthalmology. Mosby: St Louis, 1980, pp 86-119.

6 Cuero RG. Ecological distribution of Fusarium solani and its opportunistic action related to mycotic keratitis in Cali, Colombia. J Clin Microbiol 1980; 12: 455-461.

7 Liesegang TJ, Forster RK. Spectrum of microbial keratitis in South Florida. Am J Ophthalmol 1980; 90: 38-47.

8 Houang E, Lam D, Fan D, Seal D. Microbial keratitis in Hong Kong: relationship to climate, environment and contact-lens disinfection. Trans R Soc Trop Med Hyg 2001; 95 361-367.

9 Wilhelmus KR, Jones DB. Curvularia keratitis. Trans Am Ophthalmol Soc 2001; 99: 111-132.

10 Rosa Jr RH, Miller D, Alfonso EC. The changing spectrum of fungal keratitis in South Florida. Ophthalmology 1994; 101: 1005-1013.

11 Hagan M, Wright E, Newman M, Dolin P, Johnson G. Causes of suppurative keratitis in Ghana. Br J Ophthalmol 1995; 79: 1024-1028.

12 Nelson PE, Dignani MC, Anaissie EJ. Taxonomy, biology and clinical aspects of Fusarium species. Clin Microbiol Rev 1994; 7: 479-504.

13 Thomas PA. Tropical ophthalmomycoses. In: Seal DV, Bron AJ, Hay J (eds). Ocular Infection: Investigation and Treatment in Practice. Martin Dunitz: London, 1998, pp 121-142.

14 Gopinathan U, Garg P, Fernandes M, Sharma S, Athmanathan S, Rao GN. The epidemiological features and laboratory results of fungal keratitis: a 10-year review at a referral eye care center in South India. Cornea 2002; 21: 555-559.

15 Wilson LA, Ajello L. Agents of oculomycosis: fungal infections of the eye. In: Collier L, Balows A, Sussman A (eds). Topley and Wilson's Microbiology and Microbial Infections, 9th edn, Vol 4, Medical Mycology (Ajello L, Hay RJ (section eds)). Arnold: London, 1998, pp 525-567.

16 Jones BR. Principles in the management of oculomycosis. Am J Ophthalmol 1975; 79: 719-751.
17 Vemuganti GK, Garg P, Gopinathan U, Naduvilath TJ John RK, Buddi R et al. Evaluation of agent and host factors in progression of mycotic keratitis: A histologic and microbiologic study of 167 corneal buttons. Ophthalmology 2002; 109: 1538-1546.

18 Klotz SA, Penn CC, Negvesky GJ, Butrus SI. Fungal and parasitic infections of the eye. Clin Microbiol Rev 2000; 13: 662-685.

19 McLeod SD. The role of cultures in the management of ulcerative keratitis. Cornea 1997; 16: 381-382.

20 Kompa S, Langefeld S, Kirchkof B, Schrage N. Corneal biopsy in keratitis performed with the microtrephine. Graefe's Arch Clin Exp Ophthalmol 1999; 237: 915-919.

21 Alexandrakis G, Haimovici R, Miller D, Alfonso EC. Corneal biopsy in the management of progressive microbial keratitis. Am J Ophthalmol 2000; 129: 571-576.

22 Rao NA. A laboratory approach to rapid diagnosis of ocular infections and prospects for the future. Am J Ophthalmol 1989; 107: 283-291.

23 Seal DV, Bron AJ, Hay J. Ocular infection: investigation and treatment in practice. Martin Dunitz: London, 1998.

24 Thomas PA, Kuriakose T, Kirupashanker MP, Maharajan VS. Use of lactophenol cotton blue mounts of corneal scrapings as an aid to the diagnosis of mycotic keratitis. Diagn Microbiol Infect Dis 1991; 14: 219-224.

25 Ishibashi Y, Kaufman HE. Corneal biopsy in the diagnosis of keratomycosis. Am J Ophthalmol 1986; 101: 288-293.

26 Brooks JG, Coster DJ. Non-ulcerative fungal keratitis diagnosed by posterior lamellar biopsy. Aust NZ J Ophthalmol 1993; 21: 115-119.

27 Ishibashi Y, Hommura S, Matsumoto Y. Direct examination vs. culture of biopsy specimens for the diagnosis of keratomycosis. Am J Ophthalmol 1987; 103: 636-640.

28 Panda A, Mohan M, Mukherjee G. Mycotic keratitis in Indian patients. Indian J Ophthalmol 1984; 32: 311-315.

29 Robin JR, Chan R, Rao NA, Sharma S, Srinivasan M. Fluorescein-conjugated lectin visualization of fungi and acanthamoebae in infectious keratitis. Ophthalmology 1989; 96: 1198-1202.

30 Garcia ML, Herreras JM, Dios E, Argueso P, Almaraz A. Evaluation of lectin staining in the diagnosis of fungal keratitis in an experimental rabbit model. Mol Vision 2002; 8: 10-16.

31 Arora I, Singhvi S. Impression debridement of corneal lesions. Ophthalmology 1994; 101: 1935-1940.

32 Alexandrakis G, Jalali S, Gloor P. Diagnosis of Fusarium keratitis in an animal model using the polymerase chain reaction. Br J Ophthalmol 1998; 82: 306-311.

33 Ferrer C, Munoz G, Alio JL, Abad JL, Colom F. Polymerase chain reaction diagnosis in fungal keratitis caused by Alternaria alternata. Am J Ophthalmol 2002; 133: 398-399.

34 Gaudio PA, Gopinathan U, Sangwan V, Hughes TE. Polymerase chain reaction based detection of fungi in infected corneas. Br J Ophthalmol 2002; 86: 755-760.

35 National Committee for Clinical Laboratory Standards (NCCLS). Reference Method for Broth Dilution Antifungal Susceptibility Testing of Yeasts. Approved Standard M27A. NCCLS: Wayne, PA, 1997.

36 Wong T-Y, Fong K-S, Tan DT. Clinical and microbial spectrum of fungal keratitis in Singapore: a 5-year retrospective study. Int Ophthalmol 1997; 21: 127-130.

37 Kadam SP. Application of betadine (povidone-iodine) to infected corneal ulcers. Indian J Ophthalmol 1987; 35(5-6): 135-136. 
38 Fiscella RG, Moshifar M, Messick CR, Pendland SL, Chandler JW, Viana M. Polyhexamethylene biguanide (PHMB) in the treatment of experimental Fusarium keratomycosis. Cornea 1997; 16: 447-449.

39 Rehman MR, Minassian DC, Srinivasan M, Martin MJ, Johnson GJ. Trial of chlorhexidine gluconate for fungal corneal ulcers. Ophthalmic Epidemiol 1997; 4: 141-149.

40 Rehman MR, Johnson GJ, Husain R, Howlader SA, Minassian DC. Randomised trial of $0.2 \%$ chlorhexidine gluconate and $2.5 \%$ natamycin for fungal keratitis in Bangladesh. Br J Ophthalmol 1998; 82: 919-925.

41 Mohan M, Gupta SK, Kalra VK, Vajpayee RB, Sachdev MS Topical silver sulphadiazine: a new drug for ocular keratomycosis. Br J Ophthalmol 1988; 72: 192-195.

42 Johns KJ, O'Day DM. Pharmacologic management of keratomycosis. Surv Ophthalmol 1988; 33: 178-188.

43 Stern GA, Buttross M. Use of corticosteroids in combination with antimicrobial drugs in the treatment of infectious corneal disease. Ophthalmology 1991; 98: 847-853.

44 Tanure MA, Cohen EJ, Grewal S, Rapuano CJ, Laibson PR. Spectrum of fungal keratitis at Wills Eye Hospital, Philadelphia, Pennsylvania. Cornea 2000; 19: 307-312.

45 Hirose H, Terasaki H, Awaya S, Yasuma T. Treatment of fungal corneal ulcers with amphotericin B ointment. Am J Ophthalmol 1997; 124: 836-838.

46 Jones BR, Clayton YM, Oji EO. Recognition and chemotherapy of oculomycosis. Postgrad Med J 1979; 55 625-628.

47 Panda A, Sharma N, Angra SK. Topical fluconazole therapy of Candida keratitis. Cornea 1996; 15: 373-375.

48 Thomas PA, Rajasekaran J. Treatment of Aspergillus keratitis with imidazoles and related compounds. In: Vanden Bossche H, Mackenzie DWR, Cauwenbergh G (eds). Aspergillus and Aspergillosis. Plenum Press: New York, 1988, pp 267-279.

49 Thomas PA, Abraham DJ, Kalavathy CM, Rajasekaran J. Oral ketoconazole in keratomycosis. Indian J Ophthalmol 1987; 35: 197-203.
50 Thomas PA, Abraham DJ, Kalavathy CM, Rajasekaran J Oral itraconazole therapy for mycotic keratitis. Mycoses 1988; 31: 271-279.

51 Rao SK, Madhavan HN, Rao G, Padmanabhan P. Fluconazole in filamentous fungal keratitis. Cornea 1997; 16: 700.

52 Rajasekaran J, Thomas PA, Srinivasan R. Ketoconazole in keratomycosis. In: Blodi F, Brancato R, Cristini G, d'Ermo F, Esente I, Musini A et al (eds). Acta XXV Concilium Ophthalmologicum. Kugler Ghedini: Amsterdam, 1987, pp 2462-2467.

53 Rajasekaran J, Thomas PA, Kalavathy CM, Joseph PC, Abraham DJ. Itraconazole therapy for fungal keratitis. Indian J Ophthalmol 1987; 35: 157-160.

54 Thomas PA. Mycotic keratitis. J Antimicrob Chemother 1999; 44 (Suppl A): 11(14-60)

55 Alino AM, Perry HD, Kanellopoulos AJ, Donnenfeld ED, Rahn EK. Conjunctival flaps. Ophthalmology 1998; 105: 1120-1123.

56 Kuriakose T, Thomas PA. Keratomycotic malignant glaucoma. Indian J Ophthalmol 1991; 39: 118-121.

57 Thomas PA, Garrison RG, Jansen T. Intrahyphal hyphae in corneal tissue from a case of keratitis due to Lasiodiplodia theobromae. J Med Vet Mycol 1991; 29: 263-267.

58 Kiryu H, Yoshida S, Suenaga Y, Asahi M. Invasion and survival of Fusarium solani in the dexamethasone-treated cornea of rabbits. J Med Vet Mycol 1991; 29: 395-406.

59 Zhu WS, Wojdyla K, Donlon K, Thomas PA, Eberle HI. Extracellular proteases of Aspergillus flavus. Diagn Microbiol Infect Dis 1990; 13: 491-497.

60 Raza SK, Mallett AI, Howell SA, Thomas PA. An in vitro study of the sterol content and toxin production of Fusarium isolates from mycotic keratitis. J Med Microbiol 1994; 41: 204-208.

61 Gopinathan U, Ramakrishna T, Willcox M, Rao CM, Balasubramanian D, Kulkarni A et al. Enzymatic clinical, and histologic evaluation of corneal tissues in experimental fungal keratitis in rabbits. Exp Eye Res 2001; 72: 433-442. 